Itinéraires Itinéraires

Littérature, textes, cultures

2010-3 | 2010

Médiévalisme

\title{
Isabelle Pantin, Tolkien et ses légendes. Une expérience en fiction
}

Paris, CNRS Éditions, coll. « Médiévalisme(s) », 2009, 320 p.

\section{Michèle Gally}

\section{(2) OpenEdition}

\section{Journals}

Édition électronique

URL : http://journals.openedition.org/itineraires/1911

DOI : 10.4000/itineraires. 1911

ISSN : 2427-920X

Éditeur

Pléiade

Édition imprimée

Date de publication : 1 novembre 2010

Pagination : 189-191

ISBN : 978-2-296-13150-7

ISSN : 2100-1340

Référence électronique

Michèle Gally, «Isabelle Pantin, Tolkien et ses légendes. Une expérience en fiction », Itinéraires [En ligne], 2010-3 | 2010, mis en ligne le 01 novembre 2010, consulté le 22 septembre 2020. URL : http:// journals.openedition.org/itineraires/1911 ; DOI : https://doi.org/10.4000/itineraires.1911

Ce document a été généré automatiquement le 22 septembre 2020.

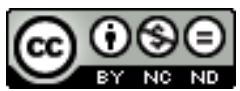

Itinéraires est mis à disposition selon les termes de la licence Creative Commons Attribution - Pas d'Utilisation Commerciale - Pas de Modification 4.0 International. 


\section{Isabelle Pantin, Tolkien et ses légendes. Une expérience en fiction}

Paris, CNRS Éditions, coll. « Médiévalisme(s)», 2009, 320 p.

\section{Michèle Gally}

\section{RÉFÉRENCE}

Isabelle Pantin, Tolkien et ses légendes. Une expérience en fiction, Paris, CNRS Éditions, coll. «Médiévalisme(s)», 2009, 320 p.

1 Isabelle Pantin, spécialiste de la littérature de la Renaissance et de l'histoire de la cosmologie, se lance dans cette étude sur d'autres temps et d'autres mondes, ceux, fascinants et souvent mal compris, de l'écrivain et universitaire anglais J.R.R Tolkien (1892-1973). Le succès mondial, relancé par le film de Peter Jackson en 2001, du Seigneur des Anneaux, œuvre phare d'un auteur plus prolixe qu'on ne croit, ne laisse pas d'être ambigu. Au nombre immense de ses amateurs, parfois fanatiques, s'oppose la réserve de spécialistes de la littérature du $\mathrm{xx}^{\mathrm{e}}$ siècle, hésitant à le consacrer comme un écrivain contemporain important.

2 C'est à partir de ce paradoxe qu'a voulu travailler Isabelle Pantin : il lui a semblé que ce contraste entre succès de librairie et hésitation de la critique universitaire permettait, au-delà de ce cas, de réfléchir sur la définition de la littérature, de la création littéraire et des critères permettant de distinguer grande littérature et œuvres mineures. N'étant ni médiéviste (comme Tolkien), ni vingtiémiste, Isabelle Pantin s'est aussi sentie libre de jouer à la fois de son savoir théorique et de son approche savante de la littérature et de sa réception de lectrice enthousiaste de Tolkien.

C'est peut-être cette position mixte qui fait la valeur et l'intérêt de son étude. Son projet est de montrer d'abord l'expérience de fiction, plus exactement "en fiction » (sous-titre de l'ouvrage), qu'a été la démarche créatrice de Tolkien toute sa vie à travers la production de multiples textes souvent inaboutis, inachevés, réécrits de diverses manières. Tolkien, en effet, n'est pas l'homme d'un seul livre ou même de deux (avec 
Bilbo le Hobbit) mais d'un très grand nombre de récits et « légendes » dont Le Seigneur des Anneaux représente sans doute l'acmé et une forme de résolution sans en être le total achèvement. L'œuvre se présente, en effet, en constant devenir et ne se conclut pas sur une image idyllique pas plus qu'elle ne sacrifie à la représentation d'un âge d'or originel (Le Silmarillion). En cela, et en dépit des apparences, elle appartient, à sa manière, à son époque.

4 Pour nous guider dans ce labyrinthe de textes dont elle parcourt toute l'étendue, et en approcher le créateur, Isabelle Pantin engage d'abord une quête de l'homme Tolkien, difficile à cerner, et procède par cercles concentriques. Les relations de l'auteur à l'histoire de son temps, qu'il n'a pas reniée, participant à la guerre de 1914-1918 et à travers ses fils à celle de 1939-1945, sont cependant à déceler plus à travers ses textes que par des confidences dont il fut très avare.

5 Pour dessiner une biographie intellectuelle, il faut donc faire le détour par les amis de Tolkien, le milieu des professeurs d'Oxford, supposer des échanges réciproques, pister des rapprochements poétiques et philosophiques (dont Bergson?). L'œuvre, foisonnante et multiple, apparaît, en effet, comme le point non seulement focal mais unique auquel le critique peut se raccrocher. L'œuvre de Tolkien serait bien « consubstantielle » à son auteur, un peu à la manière de celle de Montaigne !

6 L'enquête à laquelle nous convie minutieusement Isabelle Pantin est un peu aride au départ. Son souci de contextualisation lui fait déployer de nombreuses références, et composer des ébauches de monographies de divers auteurs au risque pour le lecteur de s'y perdre et de perdre de vue l'œuvre propre de Tolkien. Procédant comme avec les textes scientifiques $\mathrm{du} \mathrm{xvI} \mathrm{e}^{\mathrm{e}}$ siècle dont elle est une spécialiste, elle constitue un dossier complet d'influences qui par moments devient vertigineux dans le désir qui l'anime de ne négliger aucune rencontre possible, de rendre compte de toutes les hypothèses.

7 Peu à peu cependant le charme opère au fur et à mesure que l'on pénètre dans ces textes inclassables selon des normes génériques, problématiques pour une approche littéraire de la "modernité ", décevants aussi pour qui voudrait y retrouver plus qu'un parfum de Moyen Âge, enfin irréductibles simplement à la fantasy, dont on a fait de Tolkien le créateur (à tort) ou le parangon. Ces textes, fascinants et séduisants au sens propre, mettent donc mal à l'aise le spécialiste du roman comme celui des contes, car ils ne sont rien de tout cela et tout cela quand même. C'est ce nœud, irritant ou excitant comme on le voudra, qu'Isabelle Pantin affronte avec ses armes d'universitaire. Mais se tenant du côté de Tolkien, pleine d'admiration pour sa force inventive et recréative, elle met constamment en question le clivage vraie/fausse littérature (ou petite/grande littérature) d'où elle était partie, pour finalement prouver son inanité.

Le fil conducteur de l'espace/temps autour duquel se construisent les derniers chapitres semble, en particulier, d'une grande pertinence pour comprendre l'œuvre et pour comprendre la démarche du critique. Il aide à la fois à relier toutes les légendes inventées par l'auteur et à lire en vertu de leur organisation globale les récits courts ou longs comme Le Seigneur des Anneaux. Les pages sur la cartographie tolkienienne, et l'imaginaire de l'espace dont elle témoigne, convainquent de l'ambition de créer pleinement un monde imaginaire (y compris dans sa dimension cosmologique) qui animait l'auteur et dans lequel il s'est lui-même en grande partie immergé. L'étude aboutit à démontrer la cohérence d'une représentation qui passe par l'invention d'une mythologie au second degré. Ainsi l'analyse du Silmarillion et du monde des «Premiers Âges» montre comment se mettent en place tous les éléments d'un univers 
mythologique: histoire, cosmographie, langues, transmission. Là me paraît être le principal intérêt de cette étude. On y cherche non pas l'appartenance de Tolkien à tel ou tel genre mais on y perçoit le travail (fou ?) d'une invention de mythes, l'élaboration d'une écriture mimétique de celle que les médiévaux accomplirent à partir des traces laissées dans leur mémoire poétique de fragments mythologiques païens (celtes, germaniques, etc.) qu'ils combinèrent avec la pensée chrétienne et qu'ils ne cessèrent de refaçonner et de reformuler, du vers à la prose, du récit bref à la somme romanesque. S'expriment à plusieurs reprises, chez Tolkien, une interrogation sur les problèmes de la médiation et une réflexion sur les liens entre le mythe et l'histoire.

Car le plus fascinant chez lui, tel qu'il ressort de cette étude, est bien sa volonté de constituer des légendes pour ainsi dire en trompe-l'œil. C'est pourquoi on trouve à de nombreux moments une mise en abyme de l'écriture des textes mémoriels: Bilbo le Hobbit fait des traductions des livres elfiques, son journal et celui de Frodo sont copiés, complétés et conservés, constituant trois « traditions » pour le nouveau Gondor.

Enfin, la création pour lui, comme le dit et le démontre Isabelle Pantin, a un caractère fondamentalement évolutif. Il le représente dans son œuvre et le vit en tant qu'écrivain. Il émarge en cela à une certaine conception de la littérature comme l'invention d'histoires, leur transformation, leur oubli et leur renaissance. Pour Isabelle Pantin, il y a là une " image émouvante de ce qu'est la littérature au cours des temps » (p. 160).

11 Il est permis de ne pas la suivre totalement sur cette définition mais son livre complète, au sein des trop rares études en français sur Tolkien, l'ouvrage de Vincent Ferré, Tolkien: Sur les rivages de la terre du milieu (Christian Bourgois, 2001) qui en était pionnier. L'importance des informations qu'elle nous donne, et surtout son parti-pris (courageux!) de proposer une lecture globale des textes de Tolkien et de resituer Le Seigneur des Anneaux en relation avec les récits qui l'ont nourri plus que seulement préparé, rendent son étude indispensable à lire pour tous ceux qui s'intéressent aux processus d'invention de l'univers de Tolkien et pas seulement à son imagerie que le succès du film a renforcée au mépris du travail d'écriture de l'auteur.

\section{AUTEURS}

\section{MICHÈLE GALLY}

Université de Provence - GDR «Théories du poétique » 